\title{
Conformational gating in ammonia lyases
}

2 Matteo Lambrughi ${ }^{1}$, Željka Sanader Maršić ${ }^{1}$, Veronica Saez-Jimenez ${ }^{1,2}$, Valeria Mapelli ${ }^{2}$, Lisbeth

3 Olsson $^{2}$, Elena Papaleo ${ }^{1,3 *}$

4

51 Computational Biology Laboratory, Danish Cancer Society Research Center, Copenhagen,

6 Denmark

$7{ }^{2}$ Division of Industrial Biotechnology, Department of Biology and Biological Engineering, Chalmers

8 University of Technology, Göteborg, Sweden

$9{ }^{3}$ Translational Disease Systems Biology, Faculty of Health and Medical Sciences, Novo Nordisk

10 Foundation Center for Protein Research University of Copenhagen, Copenhagen, Denmark

Corresponding author: Elena Papaleo, elenap@cancer.dk

ABSTRACT

15 Ammonia lyases $(A L)$ are enzymes of industrial and biomedical interest. Knowledge

16 of $A L$ structure-dynamics-function relationship would be instrumental for making use

17 of the application potential of these enzymes. We investigated, using microsecond

18 molecular dynamics, the conformational changes in the proximity of the catalytic

19 pocket of a 3-methylaspartate ammonia lyase (MAL) as a model system. In

20 particular, we identified two regulatory elements in the MAL structure, i.e., the $\beta 5-\alpha 2$

21 loop, and the helix-hairpin-loop subdomain. We showed that they undergo

22 conformational changes switching from 'occluded' to 'open' states. We observed that

23 these rearrangements are coupled to changes in the accessibility of the active site.

24 The $\beta 5-\alpha 2$ loop and the helix-hairpin-loop subdomain modulate the formation of

25 tunnels from the protein surface to the substrate binding site, making the active site

26 more accessible to the substrate when they are in an open state. We pinpointed a

27 sequential mechanism, in which the helix-hairpin-loop subdomain needs to break a

28 subset of intramolecular interactions first, to then allow the opening of the $\beta 5-\alpha 2$ loop

29 and, as a consequence, make the $\mathrm{AL}$ catalytic pocket accessible for the substrate. 
1 Our data suggest that protein dynamics need to be considered in the design of new

$2 \quad A L$ variants for protein engineering and therapeutic purposes.

3 Keywords

4 molecular dynamics simulations, conformational changes, disordered regions,

5 conformational switches, correlated motions

6

7 Introduction

8 Ammonia lyases (ALs) are a broad class of enzymes which catalyze different

9 transformations based on $\alpha$ - and $\beta$-amino acid scaffolds, such as the deamination

10 and isomerization of natural amino acids through the reversible cleavage or the

11 shifting of a C-N bond. ALs are highly heterogeneous in their structures and

12 mechanisms of action, as attested by the fact that they cover 31 diverse EC sub-

13 classes and they are characterized by a high stereoselectivity for their substrates [1].

14 These enzymes are commercially appealing for their industrial [2,3] and biomedical

15 applications [1,2]. For example, they have been suggested as potential cancer

16 biotherapeutics [1] due to the fact ALs could prevent the supply of the tumor cells

17 with essential metabolites. One example is the histidine ammonia lyase which

18 influences the growth of ovarian and prostate cancer cells by histidine deamination

19 [1]. The deamination reaction produces urocanic acid and ammonia, eliminating

20 histidine as building block and contributes to preventing protein synthesis, which is

21 essential for the growth of cancer cells. The catalytic mechanism of ALs is well [3-9].

22 Among them, 3-methylaspartate ammonia lyases, or methylaspartases (MALs) [3]

23 catalyze the reversible deamination of 3-methylaspartate to mesaconate. MALs

24 belong to the enolase superfamily and they catalyze a broader range of reactions

25 than other ammonia lyases [1] They are potentially versatile and a promising target 
1 to design new variants with different substrate specificity or improved activity and

2 stability. For example, the MAL variant isolated from Citrobacter amalonaticus

3 (CaMAL) has been engineered for enantionselective synthesis of $\mathrm{N}$-substituted

4 aspartic acids, which are essential building blocks for pharmaceutical, artificial

5 sweeteners, synthetic enzymes and peptidomimetics [10,11].

$6 \mathrm{MAL}$ is a dimeric enzyme. Only a few crystallographic three-dimensional (3D)

7 structures of the dimeric form of MALs are available, deposited in the Protein Data

8 Bank (PDB), as the entries $1 \mathrm{KKO}, 1 \mathrm{KKR}$ [12], $1 \mathrm{KD} 0,1 \mathrm{KCZ}$ [13], along with two

9 mutated variants with PDB entries $3 Z \mathrm{VH}, 3 Z \mathrm{VI}$ [11]. CaMAL is a homodimeric

10 enzyme where each monomer (here referred to as $A$ and $B$ ) is composed by 826

11 amino acids and can be divided into two domains: an N-terminal (residues 1-160)

12 and a C-terminal domain (residues 170-413) connected by $\beta$-sheet regions (Figures

13 1A-B). The C-terminal domain folds into a triosephosphate-isomerase (TIM) barrel

14 structure, consisting of eight $\alpha$-helices and eight parallel $\beta$-strands, while the $\mathrm{N}$ -

15 terminal domain is composed of a three antiparallel $\beta$-strands and four $\alpha$-helices

16 (Figure 1B).

17 The catalytic mechanism of CaMAL has been inferred from the analysis of its 3D

18 structure, the comparison with other members of the enolase family and validated by

19 experimental mutagenesis [14,15]. The mutagenesis studies revealed the

20 importance of specific residues on the structural integrity, activity, and regio- and

21 diastereoselectivity of CaMAL (Figure 1C). The reaction catalyzed by CaMAL

22 requires the presence of cations, generally provided by the metal magnesium $\left(\mathrm{Mg}^{2+}\right)$

23 atom in the catalytic pocket, while the residues K331, H194 and Q329 form the

24 catalytic triad (Figure 1C). K331 acts as the base catalyst, while the $\mathrm{Mg}^{2+}$ metal ion

25 and the residues $\mathrm{H} 194$ and Q329 are responsible for the stabilization of the enolate 
1 anion, and binding to the 4-carboxylate group of the substrate [14]. Additionally, Q73,

2 F170, Q172, Y356, T360, C361 and L384 interact with different functional groups of

3 the substrate and are located in the pocket (Figure 1C).

4 The N-terminal domain of CaMAL bears, in the proximity of the catalytic pocket, two

5 structural elements, that we refer as $\beta 5$ - $\alpha 2$ loop (residues 70-85) and helix-hairpin-

6 loop subdomain (hhl, residues 12-51) (Figure 1C). The $\beta 5-\alpha 2$ loop connects the

7 strand $\beta 5$ with the helix $\alpha 2$ and includes Q73, which form water-mediated hydrogen

8 bonds with the substrate and alters CaMAL $\mathrm{k}_{\text {cat }}$ when mutated [15]. The hhl

9 subdomain contains a short $\alpha$-helix (residues 19-25), a small $\beta$-hairpin ( $\beta 2$ - $\beta 3$

10 strands, residues 29-34) and the loop between the strands $\beta 3$ and $\beta 4$. The hhl

11 subdomain is involved in intermolecular interactions at the interface between the

12 monomers [12].

13 At the best of our knowledge, there is no information available on the conformational

14 changes of MALs that could be related to its function. It is a common feature of

15 several enzymes that disordered or partially structured regions of a folded protein

16 undergo conformational changes that modulate the access of the substrate to the

17 active site or allow for its formation [8,16,25,17-24]. A detailed investigation of the

18 dynamics of MALs is an essential step to understand how to engineer it for

19 applicative purposes.

20 We focused on CaMAL, presenting the first all-atom Molecular Dynamics (MD)

21 investigation of this family of enzyme and its implications for activity. Biomolecular

22 simulations are useful to describe protein functional dynamics, from local to more

23 global motions and the notions of the importance of dynamics for enzyme function is

24 well established [26-31]. This includes local changes in the proximity of the active or

25 substrate binding sites [25,32-37] up to conformational changes of large amplitude 
1 associated with the opening and closing of gating loops or domains that can

2 modulate the access to the catalytic site or the substrate binding pocket of an

3 enzyme $[16,22,38]$.

4

5 Results

6

7 CaMAL is a dimer in solution

8 To select which form of CaMAL to study with MD simulations, we investigated the

9 preferred quaternary structures of CaMAL in solution. In particular, we performed

10 Size-Exclusion Chromatography (SEC) to estimate CaMAL molecular weight (MW).

11 We used proteins with a known MW as calibration standards (see Materials and

12 Methods). The resulting partition coefficients $\left(\mathrm{K}_{\mathrm{av}}\right)$ plotted against the logarithm of

13 MW were fitted with a linear function (correlation coefficient $\approx 0.99)$ (Figure 2A). We

14 identified one single sharp peak for CaMAL, corresponding to a MW of $\approx 80.4 \mathrm{kDa}$

15 (Figure 2A), which is approximately double of the MW estimated for the monomeric

16 form of CaMAL (from its primary sequence, i.e., $45.5 \mathrm{kDa}$ ). We concluded that

17 CaMAL is mostly a homodimer when in solution in its substrate-unbound state, in

18 agreement with what previously reported [39].

19 In light of the SEC results, we focused on the CaMAL dimer in solution for our MD

20 simulations. We also collected two additional simulations of the monomers (i.e.,

21 chains $\mathrm{A}$ and $\mathrm{B}$ from the dimer structure) as a control.

23 CaMAL shows asymmetric dynamic patterns with high flexibility located at the $\beta 5-\alpha 2$

24 loop and the hhl subdomain 


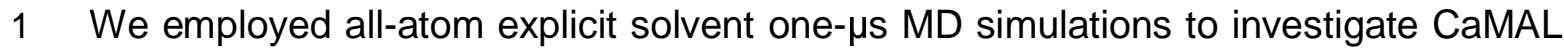

2 dynamics-structure-function relationship. In particular, we aimed at identifying

3 conformational changes that modulate the accessibility of the active site. We

4 calculated the average per-residue Root Mean Square Fluctuation (RMSF) of the Ca

5 atoms for each monomer of the CaMAL dimer as a measure of protein flexibility. The

6 peaks in the RMSF profile indicate the protein regions interested by the highest

7 flexibility (Figure 2B). We observed marked fluctuations in the $\beta 5-\alpha 2$ loop and the

$8 \mathrm{hhl}$ subdomains in both the monomeric and dimeric states of CaMAL. We found

9 similar fluctuations also in a MD simulation performed starting from another

10 crystallographic structure of CaMAL dimer (PDB entry $1 \mathrm{KK} 0$ ), supporting our findings

11 (see Github repository for more details).

12 The simulations performed on each separated monomer show higher flexibility in the

13 hhl subdomain in the case of the monomer $A$ than in the dimer, while in the

14 monomer B this region is more rigid (Figure S1). These differences suggest an

15 asymmetry in the structure and dynamics of the two monomeric subunits of CaMAL,

16 a trait common to other dimeric enzymes [40-43] (see GitHub repository for more

17 details). This asymmetry is observed for both the hhl subdomain and the $\beta 5-\alpha 2$ loop,

18 indicating that the dynamic patterns of these regions, in the dimer, are modulated by

19 the presence of the partner monomer. The RMSF estimated by our MD simulations

20 is also in agreement with the crystallographic B-factor values associated with the

21 experimental structures of the CaMAL dimer in its substrate-bound (PDB entry

$221 \mathrm{KKR}$ ) or -unbound (PDB entry 1KKO) states (Figure 2B).

24 The $\beta 5-\alpha 2$ loop and the hhl subdomain populates 'open' and 'closed' states in the $\mu$ s 25 timescale 
1 Dimensionality reduction techniques help to identify the most important

2 conformational transitions in a MD ensemble and compare simulations [44-47]. In

3 particular, we employed Principal Component Analysis (PCA) [48] and focused on

4 the 'essential subspace' described by the first two principal components (PCs)

5 (Figure 2C-D and Figure S2). We identified two main conformational states,

6 associated with conformational transitions of the $\beta 5-\alpha 2$ loop and the hhl subdomain

7 (Figure 2 C). The two regions can populate 'open' or 'occluded/closed' conformations

8 with respect to the active site (Figure $2 \mathrm{C}$ ) with a progressive opening during the

9 simulation time (Figure 2D, color gradient).

10 We observed a different dynamic pattern for the isolated monomer $\mathrm{A}$ with a large

11 exploration of the PCA subspace during the simulation time (Figure 2D, orange

12 gradient), which is related to its enhanced flexibility (Figure S1). On the contrary,

13 CaMAL remains in its closed state in the MD simulations of the isolated monomer $\mathrm{B}$,

14 mostly sampling conformations around the starting structure (Figure 2D, light blue

15 gradient), in agreement with the lower RMSF observed for this trajectory (Figure

16 S1).

17 Moreover, we extended the second replicate of the CaMAL dimer to two $\mu$ since in

18 this replicate the opening of the catalytic pocket was occurring after hundreds of ns

19 (Figure 2D, blue and dark orange gradients).

20 We then analyzed more in detail the structural changes, which were associated with

21 the conformational transitions of in the $\beta 5-\alpha 2$ loop and the hhl subdomain (Figure

22 2E). The opening of the $\beta 5-\alpha 2$ loop were associated with instability of the $\beta$-hairpin

23 (residues 29-34), loss of $\beta$ structure in the $\beta 2$ and $\beta 3$ strands (approximately in $75 \%$

24 of the simulation frames) and changes to bend/coil conformations (Figure 2E). The

25 hhl subdomain was stable during the conformational transition, with the residues 19- 
125 in a stable helical conformation in all the simulations (approximately in more than

$270 \%$ of the simulation frames) (Figure 2E).

3

4 The hhl subdomain and the $\beta 5-\alpha 2$ loop modulate the accessibility of the catalytic

5 pocket of CaMAL with a sequential mechanism

6 The conformational transitions observed during CaMAL simulations involved

7 significant rearrangements of the $\beta 5-\alpha 2$ loop and hhl subdomain in comparison to

8 their position in the starting structure (Figure 3A). To understand the relationship

9 between these rearrangements and changes in other regions of CaMAL, we

10 monitored, over the simulation time, representative pairwise distances between Ca

11 atoms of each of the residues of the $\beta 5-\alpha 2$ loop or of the hhl subdomain and the Ca

12 Q329 residue, used as a reference of the pocket (Figure 3B). We then estimated the

13 pairwise Pearson correlations of the selected distances with the solvent accessibility

14 of the catalytic pocket of CaMAL, designing a 'correlogram' where only correlations

15 with a p-value lower than 0.01 are highlighted (Figure $3 \mathbf{C}$ ). We calculated the

16 solvent accessible surface area (SASA) of each of the residues of the pocket (i.e.,

17 Q73, F170, Q172, H194, Q329, K331, Y356, T360, C361 and L384), along with their

18 cumulative SASA and the total protein SASA for each monomer. The correlogram

19 turned out to be an efficient approach to investigate possible couplings between

20 changes in the residues of the $\beta 5-\alpha 2$ loop or the hhl subdomain and the accessibility

21 of the catalytic pocket of the enzyme. We observed statistically significant positive

22 correlations between the total SASA of the pocket and the distances between Q329

23 and many of the residues of the $\beta 5-\alpha 2$ loop and the hhl subdomain (Figure $3 \mathbf{C}$ ).

24 Moreover, we applied a hierarchical cluster approach on the correlation matrices and

25 we identified two main groups of structural measurements with higher positive 
1 correlations between them (Figure $3 \mathbf{C}$ ). The first group includes the distances with

2 the residues of the $\beta 5-\alpha 2$ loop and the residues $12-14,16,29-33$ and $48-51$ of the

3 hhl subdomains that are correlated with both the total SASA of the pocket and the

4 accessibility of K331 and T360. These results suggest that the opening of the $\beta 5-\alpha 2$

5 loop modulates the accessibility of the binding pocket directly, acting of the residues

6 that are located at its entrance, such as K331 and T360 (Figure 3B). The second

7 group includes the distances with the residues 17-28 and 34-47 of the hhl

8 subdomains that are correlated with the total protein SASA and the accessibility of

9 Q73. Q73 is located in the $\beta 5-\alpha 2$ loop, and our analysis suggests that the hhl

10 subdomains play a role in acting as a conformational gate for the $\beta 5-\alpha 2$ loop (see

11 below Figure 4D).

12 In addition, we observed coupling between several residues of the $\beta 5-\alpha 2$ loop and

13 the hhl subdomain (Figure $3 \mathbf{C}$ ). Since these two structural elements are localized

14 spatially close to each other in the CaMAL structure and they form intramolecular

15 contacts, we estimated if their motions are significantly coupled at the level of

16 residue-residue displacements. In particular, we calculated the residue-residue

17 correlated motions along the trajectories using Linear Mutual Information (LMI, [49]).

18 We calculated the average LMI correlation matrices over time windows of 10 and

19100 ns for the MD simulations of CaMAL dimer to assess two different timescales

20 (Figure 3D). We calculated the pairwise differences between the LMI matrices

21 calculated for each of the two monomers in the same replicate, along with the

22 differences between the LMI matrices for the monomers A of different replicates

23 (Figure 3D and Figure S3). We concluded that the LMI matrices described similar

24 patterns of correlated fluctuations in the monomers, despite their asymmetry (see

25 above) with differences in the range of $+/-0.3$. We also observed modest differences 
1 among LMI matrices averaged over different time windows. Once assessed the

2 robustness of the LMI matrices, we analyze them to identify local couplings in the

$3 \mathrm{MD}$ ensemble (Figure 3D). In particular, we observed correlations in the motions of

4 the $35-\alpha 2$ loop and hhl subdomain (Figure 3D). Moreover, we identified coupled

5 motions between these two structural elements and most of the $\mathrm{N}$-terminal domain of

6 CaMAL (i.e., residues 1-163), suggesting that the entire N-terminal domain

7 undergoes conformational changes relatively to the C-terminal domain.

8 In summary, we observed couplings between the motions of the $\beta 5-\alpha 2$ loop and the

9 hhl subdomain, along with couplings of these two regions with the accessibility of the

10 catalytic site. We also observed that the opening of the hhl subdomain occur before

11 the opening of the $\beta 5-\alpha 2$ loop in each replicate. Our results thus suggest that the hhl

12 subdomain play a role in constraining the motions of the $\beta 5-\alpha 2$ loop, acting as a

13 conformational gate.

15 The opening of the $\beta 5-\alpha 2$ loop and $\mathrm{hhl}$ subdomain shorten the tunnels from the 16 protein surface to the active site of CaMAL

17 We estimate the formation and time-evolution of tunnels towards the catalytic site

18 (Figure 4). We used the position of four residues of the active site (i.e., Q329, D238, 19 L384, Y356) as a reference point for the tunnel analysis. We calculated the evolution 20 of the solvent-accessible tunnels from the protein surface to these residues with a 21 probe radius of $1.5 \AA$, which resembled the size of a water molecule. The analysis 22 identified from eight to ten clusters of tunnels depending on the trajectory (Figure 23 4A). We calculated the time evolution of the average length and radius of the tunnels 24 in each cluster, which indicate the absence of bottlenecks when a tunnel was 25 identified (Figure 4B-C). We observed that the length of the tunnels decreases with 
1 the opening of the hhl subdomain and the $\beta 5-\alpha 2$ loop, showing that binding pocket

2 became more easily accessible (Figure 4B-C). We also performed the same

3 analysis with a probe radius of $2.0 \AA$ to better resemble the size of the natural

4 substrate (i.e., 3-methylaspartate), obtaining similar results (see Github repository).

5 In summary, our results indicate that the opening of the hhl subdomain occur first

6 (Figure 4D) and allow the $\beta 5-\alpha 2$ loop outward displacement. Moreover, their

7 concerted opening provides an efficient access to the active site, shortening the

8 protein tunnels from the protein surface to the catalytic residues.

10 Complex networks of contacts modulate the conformational changes from 'closed' to

11 'open' states of CaMAL

12 To dissect the mechanism of opening of the substrate binding pocket through the 13 conformational changes of the hhl subdomain and the $\beta 5-\alpha 2$ loop, we also used an 14 approach based on contact maps [50]. We measured pairwise intramolecular 15 contacts between residues, their persistency along the trajectory time and estimated 16 the evens of breaking and formation of the interactions. We observed that the 17 residues of the $\beta 5-\alpha 2$ loop and the hhl subdomain in the open states are generally 18 involved in a lower number of contacts at a lower persistence then in the closed 19 states (see Github repository).

20 A cluster analysis based on the RMSD between the contact maps along the 21 trajectory identified five sequential clusters (Figure 5A). The clusters allowed to 22 study the temporal evolution of the contacts along the trajectories during the 23 transition from closed to open states of the $\beta 5-\alpha 2$ loop and the hhl subdomain. We 24 estimated the percentage increase in the pairwise distances between the residues in 25 the $\beta 5$ - $\alpha 2$ loop and the hhl subdomain with the ones in the rest of the protein, 
1 comparing the average contact maps of the first and fifth cluster. We identified

2 several pairs of residues for which we observed a marked increase in the distances

3 along the simulation time. For these residues, we measured the distances with

4 respect to residues of the $\beta 5-\alpha 2$ loop (Figure 5B) and the hhl subdomain (Figure

$5 \quad 5 \mathrm{C})$, as distance networks.

6 The distance network analysis for the $\beta 5-\alpha 2$ loop (Figure 5B) showed a significant

7 increase of distances with residues at both side of the entrance of the pocket: L196,

8 N198, Y240, M389, G359-S367 (containing two catalytic residues, i.e., T360 and

9 C361) on one side and, D307-T312 and K331-D334 (containing the catalytic residue

$10 \mathrm{~K} 331$ ), on the other side. Moreover, we observed significant changes in the

11 distances with amino acids at the bottom of the loop, such as H88-190, L93, and

$12 \mathrm{H} 127-\mathrm{R} 131$, along with several residues in the hhl subdomain or its proximity, as

13 S13-Y16, D18-A22, K24, T40-T44 and E51-S54 (Figure 5B).

14 The distance network for the hhl subdomain (Figure $\mathbf{5 C}$ ) showed an increase in the

15 distances with residues around the entrance of the pocket, such as for G174, L196,

16 N198, N199, Y240, D277, E308, C361-E365 (containing the catalytic residue C361),

17 S367, A368, and K386-G390. Furthermore, significant increases in the distance

18 network are associated with the $\beta 5-\alpha 2$ loop, such as the residues D69-G78, R80,

19 L83, L85, and A86.

20 The mapping of the residues interested by changes in the distance network during

21 the opening of the hhl subdomain and the $\beta 5-\alpha 2$ loop (Figure 5D) showed that the

$22 \mathrm{hhl}$ subdomain is involved in modulating a large area of the protein, while the $\beta 5-\alpha 2$

23 loop is mostly associated with the regions around the active site. In summary, the

24 contact-based distance network analysis allowed to identify the most significant 
1 molecular changes associated with the opening of the hhl subdomain and the $\beta 5-\alpha 2$

2 loop.

\section{Discussion}

4 An essential step toward the application of enzymes to industrial processes, protein

5 engineering and biomedical treatments, is to understand their structural properties,

6 dynamics and unveil the link with enzyme function and activity. ALs have been

7 proposed as therapeutic agents against cancer, but despite their potential, low

8 stability and rapid clearance hindered their applications [1]. The development of new

9 modified variants of ALs will open up for broad applications, but it requires more

10 detailed knowledge on their structural properties and functional mechanisms. We

11 contributed to fill this gap using computational structural biology. Our study

12 highlighted two important regulatory elements for CaMAL, i.e., the $\beta 5-\alpha 2$ loop and

13 hhl subdomain. They are highly flexible and undergo conformational changes on the

$14 \mu$ s timescale, switching from closed to open states with respect to the catalytic site.

15 The hhl subdomain and the $\beta 5-\alpha 2$ loop modulate the accessibility of the catalytic

16 pocket of the enzyme, shortening the tunnels to access the active site, in a

17 sequential mechanism where $\mathrm{hhl}$ subdomain acts as a conformational gate and

18 needs to open first to promote the detachment of the $\beta 5-\alpha 2$ loop from the residues at

19 the entrance of the pocket. This mechanism might have broader relevance for ALs,

20 as attested by the fact that other similar modulators have been reported, for example

21 in aspartate, phenylalanine and histidine ammonia lyases, $[4,8,18]$ These ALs do

22 not share similarities in sequence and are structurally unrelated but, the presence of

23 loops acting as opening/closing 'clamp' above the active site pinpoint interesting

24 similarities with the molecular mechanisms that we identified in CaMAL (Figure 6). 
1 Our results also provide a framework for the future design of mutant variants of

2 CaMAL to entrap the enzyme in its closed state to experimentally validate the effect

3 of the opening/closing transitions on the enzymatic activity of CaMAL. For example,

4 one strategy to pursue could be the engineering of disulphide bridges or mutations

5 that stabilize the interaction networks here identified. A suitable area for mutation is,

6 for example, the region above the catalytic pocket in the proximity of Y240, or the

7 residues G359-S367, which are localized at the side-entrance of the pocket. Our

8 study also opens new directions to understand, at a deeper level, how the substrate

9 recognition and binding happen in CaMAL and how this is regulated by events of

10 conformational changes of the 'gate' elements promoted by the substrate.

12 Materials and Methods

13 CaMAL expression and purification

14 We retrieved the gene sequence encoding for MAL from Citrobacter amalonaticus

15 (CaMAL) (NCBI accession numbers AB005294) from the National Center for

16 Biotechnology Information database and codon optimized for expression in

17 Escherichia coli [51]. CaMAL was synthetized by GenScript (Piscataway, NJ, USA)

18 and cloned in the vector pET28b so that a HisTag was introduced in the C-terminal

19 region of the gene. The pET-28b plasmid, which contains the CaMAL gene, was

20 transformed into Escherichia coli BL21 (DE3) for protein expression. The cells

21 containing the plasmid were grown in LB media supplemented with $50 \mu \mathrm{g} / \mathrm{ml}$ of

22 kanamycin and autoinduction media with lactose [52] at $30^{\circ} \mathrm{C}$ at $180 \mathrm{rpm}$ for $16 \mathrm{~h}$.

23 We solubilized the recollected cells in $50 \mathrm{mM}$ Tris- $\mathrm{HCl}(\mathrm{pH}$ 8) with $300 \mathrm{mM} \mathrm{NaCl}$ and

$2410 \mathrm{mM}$ imidazole and stored at $-20^{\circ} \mathrm{C}$. The thawed cells were sonicated (Branson 
1 Digital Sonifier, model 250) using a maximum amplitude of $30 \%$, in 7 cycles of $30 \mathrm{~s}$

2 and centrifuged for $20 \mathrm{~min}$ at 13500 rpms. We purified the supernatants with the

3 expressed protein by immobilized metal ion affinity chromatography (IMAC), using a

4 1-ml HisTrap column (GE Healthcare, Uppsala, Sweden) and $50 \mathrm{mM}$ phosphate $\mathrm{pH}$

$58,300 \mathrm{mM} \mathrm{NaCl}, 20 \mathrm{mM}$ imidazole buffer. A gradient up to $500 \mathrm{mM}$ imidazole was

$6 \quad$ used to elute the purified protein.

8 Size exclusion chromatography experiments

9 We estimate the molecular weight (MW) of CaMAL by SEC using a Superdex 200

10 HiLoad 16/600 column (GE Healthcare, Uppsala, Sweden), 50 mM sodium

11 phosphate $\mathrm{pH} 8.0$ with $150 \mathrm{mM}$ sodium chloride as the mobile phase, a flow rate of 1

$12 \mathrm{ml} \mathrm{min}{ }^{-1}$, and UV (280 $\left.\mathrm{nm}\right)$ detection. We used blue dextran to determine the column

13 void volume (Vo), which was $43.4 \mathrm{ml}$. We selected four proteins as molecular mass

14 standards from the gel filtration calibration kit HMW (GE Healthcare, Uppsala,

15 Sweden), i.e. ovalbumin (MW 44 000), conalbumin ( $\mathrm{MW} \sim 75$ 000), aldolase (MW

$16 \sim 158000)$ and ferritin (MW 440000 ), with elution volumes of $79.8 \mathrm{~mL}, 73.4 \mathrm{~mL}$,

$17 \quad 65.6 \mathrm{~mL}$ and $53.4 \mathrm{~mL}$, respectively. The calibration curve was constructed as the

18 partition coefficient $\left(\mathrm{K}_{\mathrm{av}}\right)$ versus $\mathrm{Log}(\mathrm{MW})$. We calculated $\mathrm{K}_{\mathrm{av}}$ using the equation $\mathrm{K}_{\mathrm{av}}$

$19=\left(V_{e}-V_{0}\right) /\left(V_{t}-V_{0}\right)$, where $V_{t}$ is the total bed volume.

21 Preparation of the initial structures for MD simulations

22 We used the X-ray structure of the homodimer of CaMAL in complex with its natural

23 substrate, i.e. the 3-methyl-aspartic acid (PDB entry $1 \mathrm{KKR},[12])$ and in the unbound

24 state (PDB entry $1 \mathrm{KKO},[12])$ as starting structures for the MD simulations. We

25 removed the coordinates of the substrate from the X-ray structure of the bound state 
1 of CaMAL. We used Modeller version 9.16 [53] to replace the seleno-methionines in

2 the PDB entries with methionines and we modeled the missing residues at the $\mathrm{N}$ -

3 and C-terminal of the protein. In the re-modeling step, we restrained all the protein

4 atoms except for the two residues immediately next to each selenomethionine in the

5 amino acid sequence. We generated 200 different models and we selected the one

6 with the lowest Root Mean Square Deviation (RMSD) calculated on all the

7 methionine atoms using as a reference their original orientation in the $\mathrm{X}$-ray

8 structure. We retained three crystallographic water molecules, which have been

9 suggested to be important for the interaction between the enzyme and its substrate

10 [12].

12 MD simulations

13 We carried out all-atom MD simulations of CaMAL in explicit solvent with Gromacs

14 version 5.1.2 [54]. We collected two one- $\mu$ s MD simulations of CaMAL in its

15 monomeric form, i.e., considering the chain A and chain B separately. Moreover, we

16 run three replicates of the substrate-unbound form CaMAL dimer from the $1 \mathrm{KKR}$

17 structure, using different random seed for the simulations and one replicate starting

18 from the $1 \mathrm{KKO}$ structure to assess the reproducibility of our findings. We employed

19 the CHARMM22 force field with CMAP corrections (namely CHARMM27) [55] and

20 the TIPS3P water model [56]. We predicted the pKa of the histidine residues with

21 PROPKA 3.1 [57] and analyzed the pattern of possible hydrogen bonds for the

22 histidine imidazole nitrogens. We modelled $\mathrm{H} 351$ with the hydrogen on the $\delta$ nitrogen

23 of the ring, while all the other histidines were modelled with hydrogens on the $\varepsilon$

24 nitrogen. 
1 Dimer simulations were done using a dodecahedral box of water with a minimum

2 distance between protein and box edges of $15 \AA$ applying periodic boundary

3 conditions, while box edges of $30 \AA$ were used for monomers. We used a larger box

4 for the simulations of the monomers to avoid that possible large rearrangements in

5 their structures, which could be caused by the removal of the partner monomer,

6 created artifacts associated with periodic boundary conditions. We gradually

7 equilibrated the system through a series of energy minimization, solvent

8 equilibration, thermalization and pressurization steps using a protocol previously

9 applied to other cases of study [58,59]. In each simulation, we used a $9 \AA$ switch

10 cutoffs for Van der Waals and short-range Coulomb interactions. We employed the

11 Particle-mesh Ewald switch summation method for long-range electrostatic

12 interactions [60]. We stored the conformations every $20 \mathrm{ps}$, and we used the LINCS

13 algorithm to constrain the heavy atom bond lengths [61]. We collected two one- $\mu$ s

14 replicates and the third replicate was extended till two $\mu$ s (see Result and Discussion

15 for more details).

16 We calculate the minimum distance between the atoms of the protein and its periodic

17 images to identify potential periodic boundary artefacts. In all the monomer

18 simulations the minimal distance was always higher than $33 \AA$ and in the dimer $39 \AA$.

Principal Component Analysis

21 We used a dimensionality reduction technique based on Principal Component

22 Analysis (PCA) $[44,45,48]$ to compare the space sampled by the different MD

23 simulations. The PCA was carried out on a concatenated trajectory of the MD

24 simulations of both monomeric and dimeric states of CaMAL (isolating each

25 monomer from the dimer first) to compare them in the same essential subspace 
$1 \quad[36,47]$. We use the the atoms belonging to the secondary structure of the TIM barrel

2 (i.e., residues 177-187, 209-225, 235-238, 251-265, 270-273, 281-297, 303-306,

3 313-322, 327-330, 338-351, 355-357, 365-378, and 382-384) for the fitting. We used

4 these residues as a reference for structural superimposition since they are located in

5 the core regions of the TIM barrel domain and do not undergo significant changes in

6 the simulations. We performed the PCA calculations for different subsets of atoms of

7 CaMAL: i) all the protein residues, ii) the region 70-85 (i.e., the $\beta 5-\alpha 2$ loop), and iii)

8 the region 12-51 (i.e., the hhl subdomain) (Figure 2 and Figure S2). We focused on

9 the first two principal components, which account for approximately $43 \%$ of the

10 variance in the whole protein scan, and approximately $60 \%$ in the $\beta 5-\alpha 2$ loop and hhl

11 subdomain PCA analysis.

13 Protein flexibility

14 We calculated the per-residue Ca-atoms Root Mean Square Fluctuation (RMSF) as

15 an index of flexibility. RMSF was calculated over non-overlapping time windows of

$1610 \mathrm{~ns}$ along the trajectories and then averaged, with a protocol used for other

17 proteins [62-64].

19 Changes in secondary structures

20 We used the DSSP dictionary [65] to estimate the different classes of secondary

21 structures attained for each residue of the $\beta 5-\alpha 2$ loop and hhl subdomain. DSSP

22 defines eight classes of secondary structure elements: $3_{10}$ helix, $\alpha$-helix, $\pi$-helix, $\beta$ -

23 sheet, $\beta$-bridge, helix turn, bend and coil. For each residue of interest a persistence

24 degree of secondary structure was calculated for each class [66]. The DSSP 
1 algorithm is based on calculating the patterns of hydrogen bonds between mainchain

2 carbonyl and amide groups.

3

$4 \quad$ Pairwise distances between selected residues

5 We calculated the time series of distances between the $\mathrm{Ca}$ atom of a reference

6 residue in the binding pocket, i.e. Q329, and the Ca atoms of all the residues of the

$7 \quad \beta 5-\alpha 2$ loop and hhl subdomain.

9 Solvent accessible surface area (SASA)

10 We estimated the SASA for all the atoms of each residues of the catalytic pocket

11 which was either involved in the catalysis or in the interaction with the substrate (i.e.,

12 Q73, F170, Q172, H194, Q329, K331, Y356, T360, C361 and L384). We also

13 calculated their cumulative SASA and the total protein SASA per monomer.

15 Correlogram

16 We collected correlogram plots for each trajectory to investigate the coupling

17 between the pairwise selected distances and the different SASA measurements. We

18 used an in-house R script based on the PerformanceAnalytics, corrplot and Hmisc R

19 packages and the rcorr() R function to calculate dependencies between all the pairs

20 of variables. We measured linear dependencies using the Pearson correlation

21 coefficient as a metric for correlation estimate and we calculated the p-value

22 associated to each correlation. We derived the correlograms using the corrplot() R

23 function and a hierarchical clustering approach to rank the correlations depending on

24 the degree of couplings between each pair of measurements. We calculated for each

25 measurement (distances and accessibilities) a bivariate scatter plot with the fitted 
1 line and the correlations coefficients with the significance levels at $p$-values of 0 ,

$20.001,0.01,0.05,0.1,1$. We performed the correlogram analysis for each monomer

3 A and B, separately as derived from the replicates of the CaMAL dimer.

\section{$5 \quad$ Correlated motions}

6 We filtered the trajectories on the Ca-atoms and fitted on the Ca-atoms of the same

7 subset of residues used as a reference for the PCA calculation, i.e., belonging to the

8 secondary structure of the TIM barrel (see above). We calculated Ca LMI correlation

9 matrices as previously described $[67,68]$ using the Wordom package version 0.22

10 [69]. We then converted the Wordom outputs to the xPyder [70] compatible format

11 using the wordomdccm2dat tool. LMI values range from 0 (i.e., uncorrelated

12 motions) to 1 (i.e., fully correlated motions). We defined a cutoff of 0.5 to identify

13 significant correlations and remove spurious correlations, as previously applied to

14 other cases of study $[58,67]$. We also employed a cutoff of distance in the sequence

15 of $+/$ - three residues to filter out the correlations between residues that are next to

16 each other in the primary sequence. We used two non-overlapping time windows of

1710 and $100 \mathrm{~ns}$, respectively to estimate average LMI correlation matrices. We

18 calculated the pairwise differences between the LMI matrices calculated for each of

19 the three different dimer replicates, to identify regions in the structure with

20 similar/dissimilar correlations. We compared the LMI matrices calculated for each

21 replicate of the dimer on the monomer $\mathrm{A}$ and the monomer $\mathrm{B}$. Moreover, we

22 evaluated the differences between the LMI matrices of the monomer $A$ and the

23 monomer A of different replicate (Figure S3). We plotted LMI matrices as heatmaps

24 using an in-house R script based on the ggplot2, reshape2 and viridis $\mathrm{R}$ functions. 
1 Tunnel analyses

2 We used the CAVER Analyst 1.0 [71] to determine the optimal parameters for the

3 calculations and identification of tunnels along the MD trajectories of CaMAL. We

4 then used the standalone version of CAVER 3.02 software [72] to identify tunnels,

5 which could mediate the access to the active site of CaMAL during the MD

6 simulations. We used as a reference point for the tunnel search the position of the

7 catalytic residues of CaMAL. In particular, we used: i) the nitrogen atom in the amide

8 group of the side chain of Q329; ii) the oxygen atom of the carboxylic group of the

9 side chain of D238; iii) the carbon atom in the methyl group of the side chain of L384

10 and, iv) the oxygen atom in the hydroxyl group of the side chain of Y356. We

11 performed the tunnel calculations on 1000 representative frames along each

12 trajectory. We removed water molecules and the $\mathrm{Mg}^{2+}$ atoms before the calculation.

13 We carried out the tunnel analysis on the MD replicates of the CaMAL dimer,

14 analyzing separately the monomers $A$ and $B$. We tested different parameters to

15 perform tunnel search and clustering with CAVER, following an optimization

16 procedure previously published [72]. In particular, we selected two set of parameters.

17 The first set aimed to monitor the evolution of solvent-accessible tunnels from the

18 protein surface towards the catalytic site, where we used a probe radius of $1.5 \AA$,

19 comparable to the size of a water molecule [73], a shell radius of $5 \AA$, shell depth of

$204 \AA$, and a clustering threshold of $7 \AA$. In the second set of parameters, we used the

21 same parameters as the first set but with a probe radius of $2.0 \AA$ to resemble the

22 size of the natural substrate 3-methylaspartate. For this purpose, we used as a

23 reference the radius of gyration of 3-methylaspartate (i.e., $2.23 \AA$ ). We used for the

24 calculation the conformation of the 3-methylaspartate in the bound crystallographic

25 structure of CaMAL (PDB entry 1KKR [12]. 
1 The tunnels were clustered using the hierarchical average-link clustering method,

2 which permit to estimate the dissimilarity (i.e., the distance) of two measured tunnels,

3 dividing each tunnel pathway by sequence of $\mathrm{N}$ points starting from the reference

4 site and then evaluating their pairwise distance in their corresponding order. We

5 used PyMOL for visual inspection and graphical representation of the tunnels.

$7 \quad$ Contact-based analyses

8 For the contact-based analyses, we used the CONtact ANalysis (CONAN) software

9 [50]. CONAN permits to obtain a statistical analysis of intramolecular contacts in

10 proteins and to investigate how they evolve along the MD trajectories. We used as

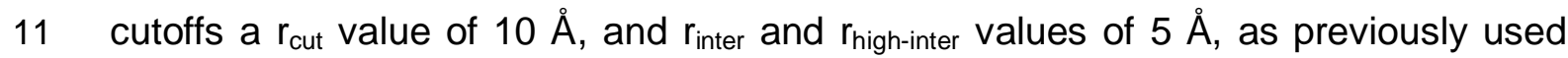

12 [50]. We performed a contact-based cluster analysis using a k-medoid clustering

13 approach based on the RMSD between the contact maps calculated for each frame

14 along each trajectory. The number of maximum clusters was set to five. We

15 observed that CONAN identifies five clusters that are sequentially distributed over

16 the simulation time. We used this analysis to study the temporal evolution of the

17 contacts along the trajectories. We calculated the average distance maps for each

18 cluster, accounting for each pair of residues over all the frames assigned to the

19 same cluster. We analyzed the average distance maps using in-house $\mathrm{R}$ scripts. We

20 calculated the percentage increase (or decrease) in the pairwise distances between

21 residues comparing the average contact maps of the first and fifth cluster. We

22 retained as significant only the pairs of residues with a percentage increase in their

23 distance higher than $50 \%$. We analyzed the data using network analysis in

24 Cytoscape. The movie of the time-evolution of the contact maps are reported in the

25 Github repository associated to the publication. 
2 Data and Software Availability

3 All the software used are freely available. The $\mathrm{R}$ and bash scripts, input files for the

4 simulations, input and output files from the modeling step, along with the results

5 generated will be soon freely available in a GitHub repository associated to our

6 publication (https://github.com/ELELAB/MAL MD). The entire MD trajectories are

7 available upon request.

9 Acknowledgements

10 The study was funded by The Swedish Research Council for Environment, 11 Agricultural Sciences and Spatial Planning (FORMAS) (project number 942-201512 1628), the NovoNordisk Foundation under the program for Biotechnology-based 13 Synthesis and Production Research (reference number NNF-170C0027588) to EP 14 and LO groups and a European Biophysical Society Association (EBSA) bursary 15 granted to VSJ to visit EP group in 2017. EP lab is part of the Center of Excellence in 16 Autophagy, Recycling and Disease (CARD), funded by the Danish National 17 Research Foundation (DNRF125) and also supported by The Danish Council for 18 Independent Research, Natural Science Research Project 1 (102517). The 19 calculations described in this paper were performed using resources provided by the 20 Swedish National Infrastructure for Computing (SNIC) at PDC Center for High 21 Performance Computing under the project SNIC 2017/1-537 and the DeiC National 22 Life Science Supercomputer at DTU.

\section{Author Contributions}


1 E.P. and M.L. designed the research; E.P. supervised the work; V.S-J. performed

2 the experiments; M.L, Z.S.M. and V.S-J. performed the modeling and simulations;

3 M.L. and Z.S.M. performed the analyses of the simulations; E.P, M.L, Z.S., V.S-J.,

4 V.M., L.O. discussed the results; M.L. and Z.S. provided scripts and code for the

5 study; M.L, Z.S. and E.P. wrote the manuscript with suggestions from all the

6 coauthors.

8 Figure Legends

Figure 1. The 3-methylaspartase ammonia lyase from Citrobacter amalonaticus

(CaMAL) has two structural elements in the proximity of the catalytic pocket,

12 i.e., the helix-hairpin-loop (hhl) and the $\beta 5-\alpha 2$ loop. A) The homodimeric structure

13 of CaMAL (PDB entry $1 \mathrm{KKR}$ ) is shown as white surface and the monomer $\mathrm{B}$ is

14 shown as cartoon for sake of clarity. The hhl subdomain and the the $\beta 5-\alpha 2$ loop are

15 in gold and brown, respectively. B) The monomer A of CaMAL dimer is shown as

16 cartoon and represented with a gradient of color from $\mathrm{N}$-terminal (brown) to C-

17 terminal (purple) with indicated the secondary structural elements in the proximity of

18 the hhl subdomain ( $\alpha 1, \beta 1-\beta 4)$ and the $\beta 5-\alpha 2$ loop ( $\alpha 2$ and $\beta 5)$. C) The residues in

19 the catalytic pocket are shown as sticks with the following color coding: Q73 red,

20 F170 orange, Q172 green, H194 forest green, Q329 aquamarine, K331 dark cyan,

21 Y356 cornflower blue, T360 navy blue, C361 purple and L384 violet red. The hhl

22 subdomain and the $\beta 5-\alpha 2$ loop are colored in gold and brown, respectively.

24 Figure 2. CaMAL is a dimer in solution with two highly flexible regions. A) Size

25 exclusion chromatography experiment shows single absorption peak at the 
1 molecular mass of $80.4 \mathrm{kDa}$ which corresponds to the dimeric form of CaMAL. B) The

2 B-factor (in the left) and RMSF (in the right) values are shown as tube representation

3 on the structure of CaMAL dimer. The thickness and the color spectrum of the tube

4 are proportional to the flexibility of the residues, with thicker red tubes representing

5 highly flexible regions and thinner blue tubes indicating more rigid regions of the

6 enzyme. The highest B-factor and RMSF values can be observed in the hhl

7 subdomain and the $\beta 5-\alpha 2$ loop. C) 2D distribution plot calculated using as reaction

8 coordinates the first and the second principal components from the PCA analysis

9 performed on all the protein atoms. We identified two densely populated subsets of

10 conformations (indicated as 'open' and 'closed' states). One representative structure

11 from each state is shown as cartoon in the lower part of the panel for illustrative

12 purposes. The 'closed' structural ensemble included conformations of the CaMAL

13 monomers similar to the initial X-ray structure. The 'open' ensemble accounted for

14 conformations of the CaMAL monomers with both the $\beta 5-\alpha 2$ loop and the hhl

15 subdomain in open states. D) We highlighted the PCA subspace sampled by each

16 trajectory. For the MD simulations of the dimer we showed the monomer $A$ and $B$

17 separately for sake of clarity. Simulations of the monomers and dimers are indicted

18 as 'mono' and 'dim', respectively and the MD replicates of the dimeric form are

19 numbered sequentially from one to three. The time evolution of each trajectory in the

20 conformational space is represented as shade of color, from dark (0 ns) to light (end

21 of the trajectory). We observed the transition from open to closed states in the dimer

22 simulations. The differences observed for the simulations of the monomer forms are

23 discussed in the text. E) We estimated the occurrence of different secondary

24 structural classes in the regions of the hhl subdomain and $\beta 5-\alpha 2$ loop during the

25 simulations. The color bars indicate the structural classes in the X-ray structure, as a 
1 reference. For sake of clarity only data obtained for the first replicate of CaMAL

2 dimer are reported. The other results are in the Github repository associated to the

3 publication.

4

5 Figure 3. The opening of the $\mathrm{hhl}$ subdomain and the $\beta 5-\alpha 2$ loop is correlated

6 with changes in the accessibility of the catalytic pocket of CaMAL. The residues

7 of the catalytic pocket are shown with the following color coding: Q73 red, F170

8 orange, Q172 green, H194 forest green, Q329 aquamarine, K331 dark cyan, Y356

9 cornflower blue, T360 navy blue, C361 purple and L384 violet red. The hhl

10 subdomain and the $\beta 5-\alpha 2$ loop are showed in gold and brown, respectively. A)

11 Surface representation of two representative structures belonging to the 'closed' (in

12 the left) and 'open' (in the right) states of the enzyme. The open conformations

13 promote a higher accessibility of the residues in the catalytic pocket. B) We showed

14 the distances between the Ca atom of Q329 in the pocket and the Ca atoms of all

15 the residues in the $\beta 5-\alpha 2$ loop and in the hhl subdomain that we used in the

16 analyses. The CaMAL is shown as white cartoon and the residues in the binding

17 pocket, for which the solvent accessibility surface area (SASA) was calculated, are

18 shown as sticks. C) The correlogram shows the Pearson correlation coefficients

19 between each pair of measurements (i.e., the selected distances and SASAs) at a p-

20 value $<0.01$. The color intensity and the size of the circle are proportional to the

21 correlation coefficients, i.e. the strength of the correlations, between each pair of

22 measurements, from dark red (negative correlations) to dark blue (positive

23 correlations). The correlogram shows a clear coupling between changes in the the

$24 \quad \beta 5-\alpha 2$ loop and in the hhl subdomain and accessibility of specific residues of the

25 catalytic binding pocket. The details are discussed in the text. D) The heatmap 
1 shows the average correlated motions in the MD trajectories, using Linear Mutual

2 Information (LMI). The LMI correlations are shown as a gradient from 0 (i.e.,

3 uncorrelated motions shown in yellow) to 1 (i.e., fully correlated motions in dark

4 blue). The LMI correlation matrix shows a coupling in the motions of the $\beta 5-\alpha 2$ loop

5 and the hhl subdomain, which are also coupled to the entire $\mathrm{N}$-terminal domain. For

6 sake of clarity, we reported the results for the monomer A of the first MD replicate of

7 the CaMAL dimeric form. The other results are in the Github repository associated to

8 the publication.

10 Figure 4. The opening of the $\beta 5-\alpha 2$ loop and hhl subdomain modulate the 11 length and the apperance of tunnels toward the active site of CaMAL. A) We

12 showed the main clusters of tunnel pathways calculated from the MD ensembles on

13 the structure of the CaMAL monomer. In particular, the tunnels calculated for each

14 conformation are shown as pathway centerlines and grouped into clusters. For each

15 conformation, if more than one tunnel pathway is grouped in the same cluster, only

16 the pathway with the lowest cost is shown. B-C) We showed, as heatmaps, the

17 temporal evolution of the two main cluster of tunnels in blue and green, respectively.

18 The plots report the average length and the radius (colors in the plot) of the tunnels

19 in the cluster for each trajectory frame. For sake of clarity, we illustrated only the

20 results for two clusters for the monomer $A$ of the first MD replicate of the CaMAL

21 dimer form. The other results are in the Github repository associated to the

22 publication. D) We showed the temporal evolution of three distances between the Ca

23 atoms of Q329 (in the pocket) and S75 (in the $\beta 5-\alpha 2$ loop, in brown), P38 and A49

24 (in the hhl subdomain, in gold and dark gold, respectively). The origin of the y-axis is

25 set to the lowest value observed for the three selected distances $(10.77 \AA)$. We 
1 showed only the results for the monomer A of the first MD replicate of the CaMAL

2 dimer form for sake of clarity. The other results are in the Github repository

3 associated to the publication.

4

5 Figure 5. Rearrangements of intramolecular contacts during the opening of the

6 hhl subdomains and the $\beta 5-\alpha 2$ loop. A) We performed a cluster analysis based on

7 the RMSD between the contact maps collected at different time points during the

8 simulation, setting the maximum number of clusters as five. The plot shows the time

9 evolution of the identified five clusters with dots colored according to the distance

10 (measured in RMSD) of each conformation from the cluster medoid, which is

11 indicated with a blue dot. B-C) Circular plots showing the pairwise distance network

12 for the $\beta 5$ - $\alpha 2$ loop (B) and the hhl subdomains (C). We estimated the percentage

13 increase of the pairwise distances between the residues of the $\beta 5-\alpha 2$ loop and the

$14 \mathrm{hhl}$ subdomain with the residues of the rest of the protein, comparing the average

15 contact maps of the first and fifth cluster. In the graphs, the edges indicate

16 percentage increase above $50 \%$ in the network and connect pairs of residues, which

17 represent the nodes. The values of percentage increase are colored as a gradient

18 from red $(50 \%)$ to purple $(300 \%)$. The node corresponding to each residue are

19 shown as points and they are colored on the base of their degree in the network,

20 from low (yellow) to high (dark blue). D) We mapped the network of residues on a

21 representative open conformation of the CaMAL dimer as green and blue sticks for

22 the $\beta 5-\alpha 2$ loop and the hhl subdomain, respectively. The hhl subdomain and the $\beta 5-$

23 a2 loop are highlighted in gold and brown, respectively and both the monomers are

24 shown. For sake of clarity, we illustrated the results for the monomer A of the first 
1 replicate of the CaMAL dimeric form. The other results are in the Github repository

2 associated to the publication.

4 Figure 6. Other ammonia lyases show conformationally heterogeneous

5 structural elements in the proximity of their catalytic sites, which could

6 undergo opening/closing transitions. We showed the structures of: i) CaMAL with

7 the hhl subdomain and the $\beta 5-\alpha 2$ loop highlighted in gold and brown, respectively; ii)

8 aspartate (AAL, PDB entry 3R6V), phenylalanine (PAL, 1W27), and histidine (HAL,

$91 \mathrm{GKM})$ ammonia lyases with their disordered regions colored in red in the proximity

10 of the catalytic site, which is indicated by a blue star.

\section{References}

13 [1] F. Parmeggiani, N.J. Weise, S.T. Ahmed, N.J. Turner, Synthetic and Therapeutic Applications of Ammonia-lyases and Aminomutases, Chem. Rev. (2017) acs.chemrev.6b00824. doi:10.1021/acs.chemrev.6b00824.

[3] M. De Villiers, V.P. Veetil, H. Raj, J. De Villiers, G.J. Poelarends, Catalytic mechanisms and biocatalytic applications of aspartate and methylaspartate

23 [4] J. Zhang, Y. Liu, A QM/MM study of the catalytic mechanism of aspartate ammonia lyases, ACS Chem. Biol. 7 (2012) 1618-1628. ammonia lyase, J. Mol. Graph. Model. 51 (2014) 113-119. doi:10.1016/j.jmgm.2014.05.003. 
1 [5] A. Heine, G. Herrmann, T. Selmer, F. Terwesten, W. Buckel, K. Reuter, High resolution crystal structure of Clostridium propionicum $\beta$-alanyl-CoA: AMmonia lyase, a new member of the "hot dog fold" protein superfamily, Proteins Struct. Funct. Bioinforma. 82 (2014) 2041-2053. doi:10.1002/prot.24557.

[6] K.T. Watts, B.N. Mijts, P.C. Lee, A.J. Manning, C. Schmidt-Dannert, Discovery of a Substrate Selectivity Switch in Tyrosine Ammonia-Lyase, a Member of the Aromatic Amino Acid Lyase Family, Chem. Biol. 13 (2006) 1317-1326. doi:10.1016/j.chembiol.2006.10.008.

[7] N.J. Turner, Ammonia lyases and aminomutases as biocatalysts for the synthesis of $\alpha$-amino and $\beta$-amino acids, Curr. Opin. Chem. Biol. 15 (2011) 234-240. doi:10.1016/j.cbpa.2010.11.009.

[8] A.L. Seff, S. Pilbák, I. Silaghi-Dumitrescu, L. Poppe, Computational investigation of the histidine ammonia-lyase reaction: A modified loop conformation and the role of the zinc(II) ion, J. Mol. Model. 17 (2011) 15511563. doi:10.1007/s00894-010-0849-7.

[9] Y. Asano, Y. Kato, C. Levy, P. Baker, D. Rice, Structure and Function of Amino Acid Ammonia-lyases, Biocatal. Biotransformation. 22 (2004) 133-140. doi:10.1080/10242420410001703496.

[10] W.J. Quax, H. Raj, P.G. Tepper, G.J. Poelarends, V. Puthan Veetil, M. de Villiers, F.J. Dekker, Enantioselective Synthesis of N-Substituted Aspartic Acids Using an Engineered Variant of Methylaspartate Ammonia Lyase, ChemCatChem. 5 (2013) 1325-1327. doi:10.1002/cctc.201200906.

[11] H. Raj, W. Szymański, J. De Villiers, H.J. Rozeboom, V.P. Veetil, C.R. Reis, M. De Villiers, F.J. Dekker, S. De Wildeman, W.J. Quax, A.M.W.H. Thunnissen, B.L. Feringa, D.B. Janssen, G.J. Poelarends, Engineering methylaspartate 
1 ammonia lyase for the asymmetric synthesis of unnatural amino acids, Nat.

$2 \quad$ Chem. 4 (2012) 478-484. doi:10.1038/nchem.1338.

3 [12] C.W. Levy, P.A. Buckley, S. Sedelnikova, Y. Kato, Y. Asano, D.W. Rice, P.J. Baker, Insights into enzyme evolution revealed by the structure of methylaspartate ammonia lyase, Structure. 10 (2002) 105-113. doi:10.1016/S0969-2126(01)00696-7.

[13] M. Asuncion, W. Blankenfeldt, J.N. Barlow, D. Gani, J.H. Naismith, The structure of 3-methylaspartase from Clostridium tetanomorphum functions via the common enolase chemical step, J. Biol. Chem. 277 (2002) 8306-8311. doi:10.1074/jbc.M111180200.

[14] H. Raj, B. Weiner, V.P. Veetil, C.R. Reis, W.J. Quax, D.B. Janssen, B.L. Feringa, G.J. Peolarends, Alteration of the diastereoselectivity of 3methlaspartate ammonia lyase by using structure-based mutagenesis, ChemBioChem. 10 (2009) 2236-2245. doi:10.1002/cbic.200900311.

[15] H. Raj, G.J. Poelarends, The roles of active site residues in the catalytic mechanism of methylaspartate ammonia-lyase, FEBS Open Bio. 3 (2013) 285-290. doi:10.1016/j.fob.2013.07.002.

[16] E. Papaleo, V. Ranzani, F. Tripodi, A. Vitriolo, C. Cirulli, P. Fantucci, L. Alberghina, M. Vanoni, L. De Gioia, P. Coccetti, An Acidic Loop and Cognate Phosphorylation Sites Define a Molecular Switch That Modulates Ubiquitin Charging Activity in Cdc34-Like Enzymes, PLoS Comput. Biol. 7 (2011). doi:10.1371/journal.pcbi.1002056.

[17] E. Papaleo, N. Casiraghi, A. Arrigoni, M. Vanoni, P. Coccetti, L. De Gioia, Loop 7 of E2 Enzymes $\square:$ An Ancestral Conserved Functional Motif Involved in the E2-Mediated Steps of the Ubiquitination Cascade, PLoS One. 7 (2012). 
1 doi:10.1371/journal.pone.0040786.

2 [18] S. Pilbák, A. Tomin, J. Rétey, L. Poppe, The essential tyrosine-containing loop

3 conformation and the role of the C-terminal multi-helix region in eukaryotic

$4 \quad$ phenylalanine ammonia-lyases, FEBS J. 273 (2006) 1004-1019.

$5 \quad$ doi:10.1111/j.1742-4658.2006.05127.x.

6 [19] R.C. Wade, M.E. Davis, B.A. Luty, J.D. Madura, J.A. McCammon, Gating of

7 the active site of triose phosphate isomerase: Brownian dynamics simulations

8 of flexible peptide loops in the enzyme., Biophys. J. 64 (1993) 9-15.

9 doi:10.1016/S0006-3495(93)81335-3.

10 [20] H.-X. Zhou, S.T. Wlodek, J.A. McCammon, Conformation gating as a

[24] A. Gora, J. Brezovsky, J. Damborsky, Gates of Enzymes, Chem. Rev. 113 mechanism for enzyme specificity, Proc. Natl. Acad. Sci. 95 (2002) 92809283. doi:10.1073/pnas.95.16.9280.

[21] M. Kokkinidis, N.M. Glykos, V.E. Fadouloglou, Protein flexibility and enzymatic catalysis, 2012. doi:10.1016/B978-0-12-398312-1.00007-X.

[22] E. Papaleo, G. Saladino, M. Lambrughi, K. Lindorff-Larsen, F.L. Gervasio, R. Nussinov, The role of protein loops and linkers in conformational dynamics and allostery., Chem. Rev. 116 (2016) 6391-6423. (2013) 5871-5923. doi:10.1021/cr300384w. doi:10.1021/acs.chemrev.5b00623.

[23] M.K. Rout, B.L. Lee, A. Lin, W. Xiao, L. Spyracopoulos, Active Site Gate Dynamics Modulate the Catalytic Activity of the Ubiquitination Enzyme E2-25K, Sci. Rep. 8 (2018) 1-15. doi:10.1038/s41598-018-25476-8.

[25] M. Pasi, L. Riccardi, P. Fantucci, L. De Gioia, E. Papaleo, Dynamic properties of a psychrophilic alpha-amylase in comparison with a mesophilic homologue., 
J. Phys. Chem. B. 113 (2009) 13585-95. doi:10.1021/jp900790n.

[26] D. Tobi, I. Bahar, Structural changes involved in protein binding correlate with intrinsic motions of proteins in the unbound state., Proc. Natl. Acad. Sci. U. S.

A. 102 (2005) 18908-13. doi:10.1073/pnas.0507603102.

[27] Z. Kurkcuoglu, A. Bakan, D. Kocaman, I. Bahar, P. Doruker, Coupling between Catalytic Loop Motions and Enzyme Global Dynamics, PLoS Comput. Biol. 8 (2012) e1002705. doi:10.1371/journal.pcbi.1002705.

[28] A. Tousignant, J.N. Pelletier, Protein motions promote catalysis, Chem. Biol. 11 (2004) 1037-1042. doi:10.1016/j.

[29] J.L. Klepeis, K. Lindorff-Larsen, R.O. Dror, D.E. Shaw, Long-timescale molecular dynamics simulations of protein structure and function., Curr. Opin. Struct. Biol. 19 (2009) 120-7. doi:10.1016/j.sbi.2009.03.004.

[30] R.O. Dror, R.M. Dirks, J.P. Grossman, H. Xu, D.E. Shaw, Biomolecular simulation: a computational microscope for molecular biology., Annu. Rev. Biophys. 41 (2012) 429-52. doi:10.1146/annurev-biophys-042910-155245.

[31] T. Saleh, C.G. Kalodimos, Enzymes at work are enzymes in motion, Science (80-. ). 355 (2017) 247-248. doi:10.1126/science.aal4632.

[32] E. Papaleo, L. Sutto, F.L. Gervasio, K. Lindorff-Larsen, Conformational changes and free energies in a proline isomerase, J. Chem. Theory Comput. 10 (2014) 4169-4174. doi:10.1021/ct500536r.

[33] R.N. Shinde, S. Karthikeyan, B. Singh, Molecular dynamics studies unravel role of conserved residues responsible for movement of ions into active site of DHBPS, Sci. Rep. 7 (2017) 1-10. doi:10.1038/srep40452.

[34] P. Mereghetti, L. Riccardi, B.O. Brandsdal, P. Fantucci, L. De Gioia, E. Papaleo, Near native-state conformational landscape of psychrophilic and 
mesophilic enzymes: probing the folding funnel model, J. Phys. Chem. B. 114 (2010) 7609-7619. doi:10.1021/jp911523h.

[35] F. del Caño-Ochoa, A. Grande-García, M. Reverte-López, M. D’Abramo, S. Ramón-Maiques, Characterization of the catalytic flexible loop in the dihydroorotase domain of the human multi-enzymatic protein CAD, J. Biol. Chem. 293 (2018) 18903-18913. doi:10.1074/jbc.RA118.005494.

[36] I. Valimberti, M. Tiberti, M. Lambrughi, B. Sarcevic, E. Papaleo, E2 superfamily of ubiquitin-conjugating enzymes: constitutively active or activated through phosphorylation in the catalytic cleft, Sci. Rep. 5 (2015) 14849. doi:10.1038/srep14849.

[37] E. Papaleo, L. Riccardi, C. Villa, P. Fantucci, L. De Gioia, Flexibility and enzymatic cold-adaptation: A comparative molecular dynamics investigation of the elastase family, Biochim. Biophys. Acta - Proteins Proteomics. 1764 (2006) 1397-1406. doi:10.1016/j.bbapap.2006.06.005.

[38] E. Papaleo, G. Renzetti, Coupled motions during dynamics reveal a tunnel toward the active site regulated by the $\mathrm{N}$-terminal $\alpha$-helix in an acylaminoacyl peptidase, J. Mol. Graph. Model. 38 (2012) 226-234. doi:10.1016/j.jmgm.2012.06.014.

[39] Y. Kato, Y. Asano, Purification and properties of crystalline 3-methylaspartase from two facultative anaerobes, Citrobacter sp. strain YG-0504 and Morganella morganii strain YG-0601., Biosci. Biotechnol. Biochem. 59 (1995) 93-9. http://www.ncbi.nlm.nih.gov/pubmed/7765982 (accessed March 15, 2019).

[40] E. Papaleo, G. Renzetti, G. Invernizzi, B. Ásgeirsson, Dynamics fingerprint and inherent asymmetric flexibility of a cold-adapted homodimeric enzyme. A case study of the Vibrio alkaline phosphatase, Biochim. Biophys. Acta - Gen. Subj. 
1

2

1830 (2013) 2970-2980. doi:10.1016/j.bbagen.2012.12.011.

[41] E. Moroni, D.A. Agard, G. Colombo, The Structural Asymmetry of Mitochondrial Hsp90 (Trap1) Determines Fine Tuning of Functional Dynamics, J. Chem. Theory Comput. 14 (2018) 1033-1044. doi:10.1021/acs.jctc.7b00766.

[42] J.M. Flynn, P. Mishra, D.N. a. Bolon, Mechanistic Asymmetry in Hsp90 Dimers, J. Mol. Biol. (2015). doi:10.1016/j.jmb.2015.03.017.

[43] T.H. Kim, P. Mehrabi, Z. Ren, A. Sljoka, C. Ing, A. Bezginov, L. Ye, R. Pomès, R.S. Prosser, E.F. Pai, The role of dimer asymmetry and protomer dynamics in enzyme catalysis, Science (80-. ). 355 (2017). doi:10.1126/science.aag2355.

[44] E. Papaleo, P. Mereghetti, P. Fantucci, R. Grandori, L. De Gioia, Free-energy landscape, principal component analysis, and structural clustering to identify representative conformations from molecular dynamics simulations: The myoglobin case, J. Mol. Graph. Model. 27 (2009) 889-899. doi:10.1016/j.jmgm.2009.01.006.

[45] I. Daidone, A. Amadei, Essential dynamics: foundation and applications, Wiley Interdiscip. Rev. Comput. Mol. Sci. 2 (2012) 762-770. doi:10.1002/wcms.1099.

[46] M. Tiberti, E. Papaleo, T. Bengtsen, W. Boomsma, K. Lindorff-Larsen, ENCORE: software for quantitative ensemble comparison, PLoS Comput. Biol. $11(2015)$ e1004415.

[47] F. Martín-García, E. Papaleo, P. Gomez-Puertas, W. Boomsma, K. LindorffLarsen, Comparing molecular dynamics force fields in the essential subspace., PLoS One. 10 (2015) e0121114. doi:10.1371/journal.pone.0121114.

[48] A. Amadei, A.B. Linssen, H.J. Berendsen, Essential dynamics of proteins., Proteins. 17 (1993) 412-25. doi:10.1002/prot.340170408. 
1 [49] O.F. Lange, H. Grubmüller, Generalized correlation for biomolecular dynamics, Proteins Struct. Funct. Bioinforma. 62 (2005) 1053-1061. doi:10.1002/prot.20784.

[50] D. Mercadante, F. Gräter, C. Daday, CONAN: A Tool to Decode Dynamical Information from Molecular Interaction Maps, Biophys. J. 114 (2018) 12671273. doi:10.1016/j.bpj.2018.01.033.

[51] P. Puigbò, E. Guzmán, A. Romeu, S. Garcia-Vallvé, OPTIMIZER: a web server for optimizing the codon usage of DNA sequences., Nucleic Acids Res. 35 (2007) W126-31. doi:10.1093/nar/gkm219.

[52] F.W. Studier, Protein production by auto-induction in high-density shaking cultures, Protein Expr. Purif. 41 (2005) 207-234. doi:10.1016/J.PEP.2005.01.016.

[53] N. Eswar, B. Webb, M. a Marti-Renom, M.S. Madhusudhan, D. Eramian, M.-Y. Shen, U. Pieper, A. Sali, Comparative protein structure modeling using MODELLER., Curr. Protoc. Protein Sci. Chapter 2 (2007) Unit 2.9. doi:10.1002/0471140864.ps0209s50.

[54] M.J. Abraham, T. Murtola, R. Schulz, S. Páll, J.C. Smith, B. Hess, E. Lindahl, GROMACS: High performance molecular simulations through multi-level parallelism from laptops to supercomputers, SoftwareX. 2 (2015) 19-25. doi:10.1016/j.softx.2015.06.001.

[55] P. Bjelkmar, P. Larsson, M.A. Cuendet, B. Hess, E. Lindahl, Implementation of the CHARMM force field in GROMACS: analysis of protein stability effects from correction Maps, virtual interaction sites, and water models., J. Chem. Theory Comput. 6 (2010) 459-66. doi:10.1021/ct900549r.

[56] W.L. Jorgensen, J. Chandrasekhar, J.D. Madura, R.W. Impey, M.L. Klein, 
Comparison of simple potential functions for simulating liquid water, J. Chem. Phys. 79 (1983) 926. doi:10.1063/1.445869.

[57] M.H.M. Olsson, C.R. Søndergaard, M. Rostkowski, J.H. Jensen, PROPKA3: Consistent Treatment of Internal and Surface Residues in Empirical $\mathrm{p} \mathrm{K}_{\mathrm{a}}$ Predictions, J. Chem. Theory Comput. 7 (2011) 525-537. doi:10.1021/ct100578z.

[58] M. Tiberti, G. Invernizzi, E. Papaleo, (Dis)similarity Index To Compare Correlated Motions in Molecular Simulations, J. Chem. Theory Comput. 11 (2015) 4404-4414. doi:10.1021/acs.jctc.5b00512.

[59] M. Nygaard, T. Terkelsen, A.V. Olsen, V. Sora, J. Salamanca, F. Rizza, S. Bergstrand, M. Di Marco, M. Vistesen, M. Lambrughi, M. Jaattela, T. Kallunki, E. Papaleo, The mutational landscape of the oncogenic MZF1 SCAN domain in cancer, Front. Mol. Biosci. 3 (2016) 1-18. doi:10.3389/fmolb.2016.00078.

[60] U. Essmann, L. Perera, M.L. Berkowitz, T. Darden, H. Lee, L.G. Pedersen, A smooth particle mesh Ewald method, J. Chem. Phys. 103 (1995) 8577. doi:10.1063/1.470117.

[61] B. Hess, H. Bekker, H. Berendsen, J. Fraaije, LINCS: A linear constraint solver for molecular simulations, J. Comput. Chem. 12 (1993) 1463-1472. doi:10.1002/(SICI)1096-987X(199709)18:12\&lt;1463::AID-JCC4\&gt;3.0.CO;2$\mathrm{H}$.

[62] K.R. Óskarsson, M. Nygaard, B. Ellertsson, S.H. Thorbjarnardottir, E. Papaleo, M.M. Kristjánsson, A single mutation Gln142Lys doubles the catalytic activity of VPR, a cold adapted subtilisin-like serine proteinase, Biochim. Biophys. Acta - Proteins Proteomics. 1864 (2016) 1436-1443. doi:10.1016/j.bbapap.2016.07.003. 
1 [63] A. Di Rita, A. Peschiaroli, P. D’Acunzo, D. Strobbe, Z. Hu, J. Gruber, M. Nygaard, M. Lambrughi, G. Melino, E. Papaleo, J. Dengjel, S. El Alaoui, M. Campanella, V. Dötsch, V. V. Rogov, F. Strappazzon, F. Cecconi, HUWE1 E3 ligase promotes PINK1/PARKIN-independent mitophagy by regulating AMBRA1 activation via IKKa, Nat. Commun. 9 (2018) 3755. doi:10.1038/s41467-018-05722-3.

[64] D. Michetti, B.O. Brandsdal, D. Bon, G.V. Isaksen, M. Tiberti, E. Papaleo, A comparative study of cold- and warm-adapted Endonucleases A using sequence analyses and molecular dynamics simulations, PLoS One. 12 (2017)

[65] W. Kabsch, C. Sander, Dictionary of protein secondary structure: Pattern recognition of hydrogen-bonded and geometrical features, Biopolymers. 22 (1983) 2577-2637. doi:10.1002/bip.360221211.

[66] E. Papaleo, M. Pasi, M. Tiberti, L. De Gioia, Molecular dynamics of mesophiliclike mutants of a cold-adapted enzyme: insights into distal effects induced by the mutations., PLoS One. 6 (2011) e24214. doi:10.1371/journal.pone.0024214.

[67] G. Invernizzi, M. Tiberti, M. Lambrughi, K. Lindorff-Larsen, E. Papaleo, Communication Routes in ARID Domains between Distal Residues in Helix 5 and the DNA-Binding Loops, PLoS Comput. Biol. 10 (2014) e1003744. doi:10.1371/journal.pcbi.1003744.

[68] O.F. Lange, H. Grubmüller, Full correlation analysis of conformational protein dynamics., Proteins. 70 (2008) 1294-312. doi:10.1002/prot.21618.

[69] M. Seeber, A. Felline, F. Raimondi, S. Muff, R. Friedman, F. Rao, A. Caflisch, F. Fanelli, Wordom: a user-friendly program for the analysis of molecular 
structures, trajectories, and free energy surfaces., J. Comput. Chem. 32 (2011) 1183-94. doi:10.1002/jcc.21688.

[70] M. Pasi, M. Tiberti, A. Arrigoni, E. Papaleo, xPyder: a PyMOL plugin to analyze coupled residues and their networks in protein structures., J. Chem. Inf. Model. 279 (2012) 1-6. doi:10.1021/ci300213c.

[71] B. Kozlikova, E. Sebestova, V. Sustr, J. Brezovsky, O. Strnad, L. Daniel, D. Bednar, A. Pavelka, M. Manak, M. Bezdeka, P. Benes, M. Kotry, A. Gora, J. Damborsky, J. Sochor, CAVER Analyst 1.0: graphic tool for interactive visualization and analysis of tunnels and channels in protein structures, Bioinformatics. 30 (2014) 2684-2685. doi:10.1093/bioinformatics/btu364.

[72] E. Chovancova, A. Pavelka, P. Benes, O. Strnad, J. Brezovsky, B. Kozlikova, A. Gora, V. Sustr, M. Klvana, P. Medek, L. Biedermannova, J. Sochor, J. Damborsky, CAVER 3.0: A Tool for the Analysis of Transport Pathways in Dynamic Protein Structures, PLoS Comput. Biol. 8 (2012) e1002708. doi:10.1371/journal.pcbi.1002708.

[73] M. Manak, M. Zemek, J. Szkandera, I. Kolingerova, E. Papaleo, M. Lambrughi, Hybrid Voronoi diagrams, their computation and reduction for applications in computational biochemistry, J. Mol. Graph. Model. 74 (2017) 225-233. doi:10.1016/j.jmgm.2017.03.018. 


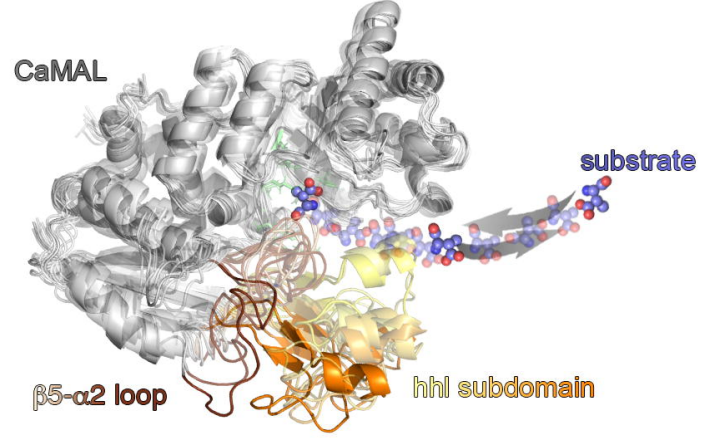


A $\quad$ h
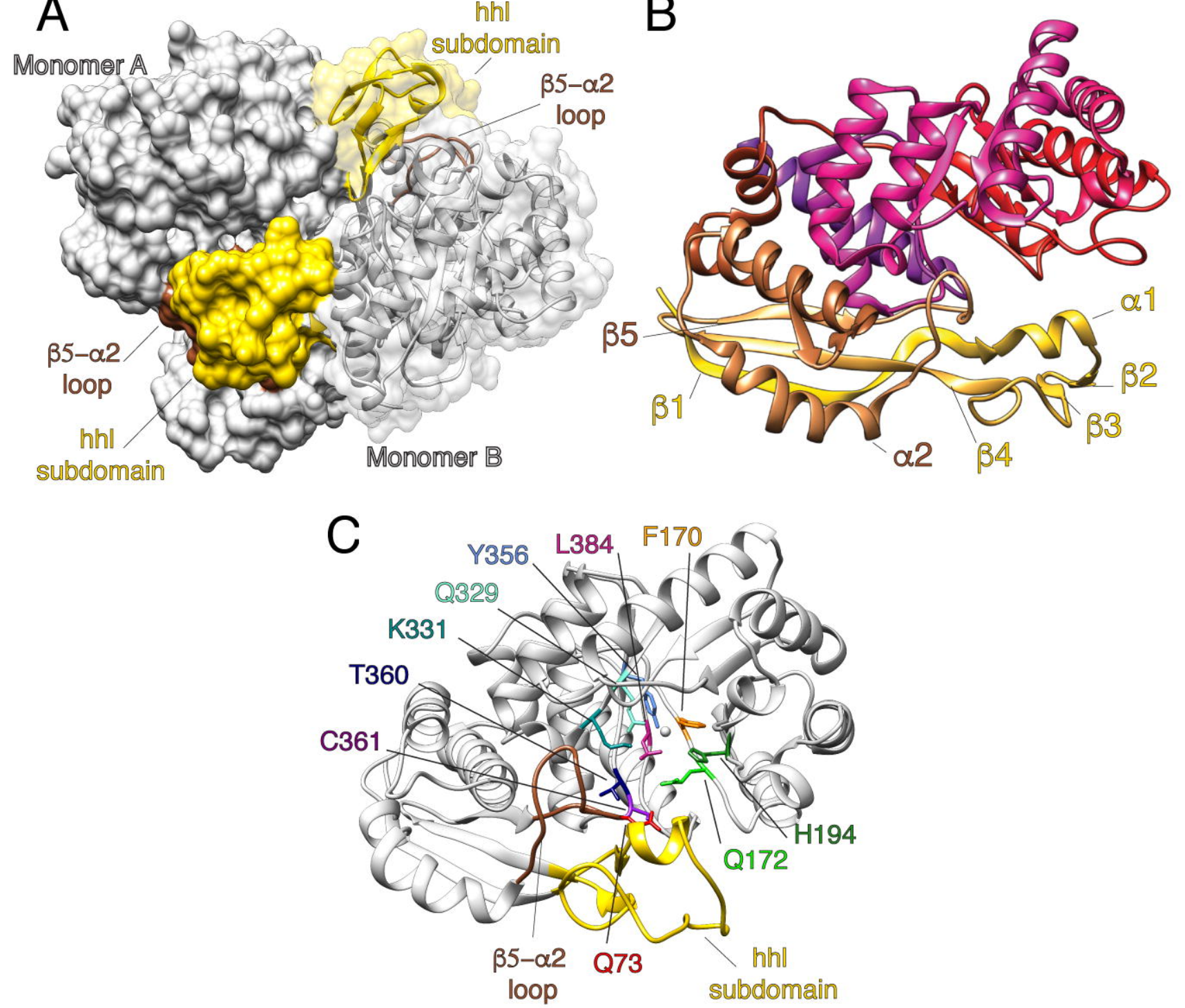


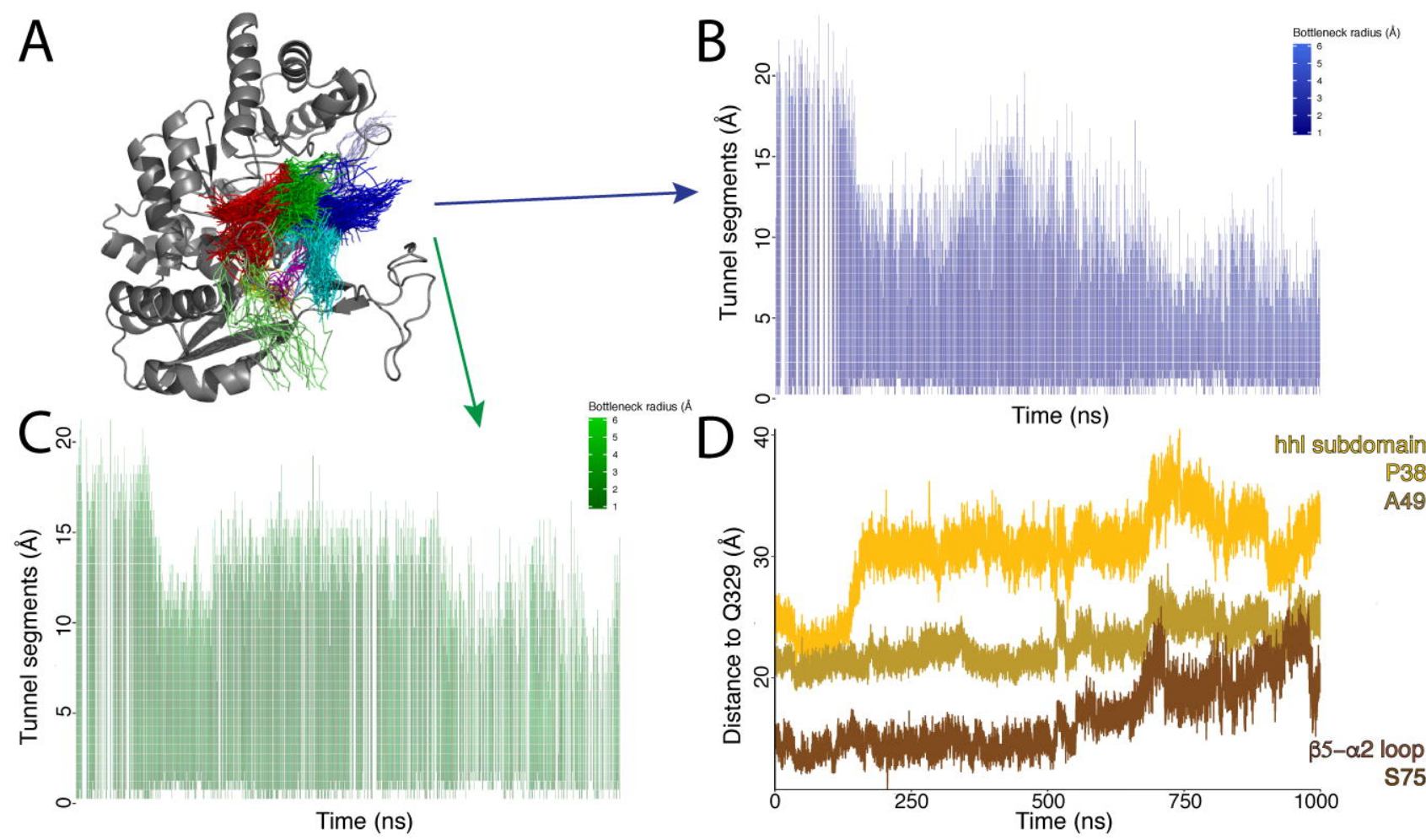


A

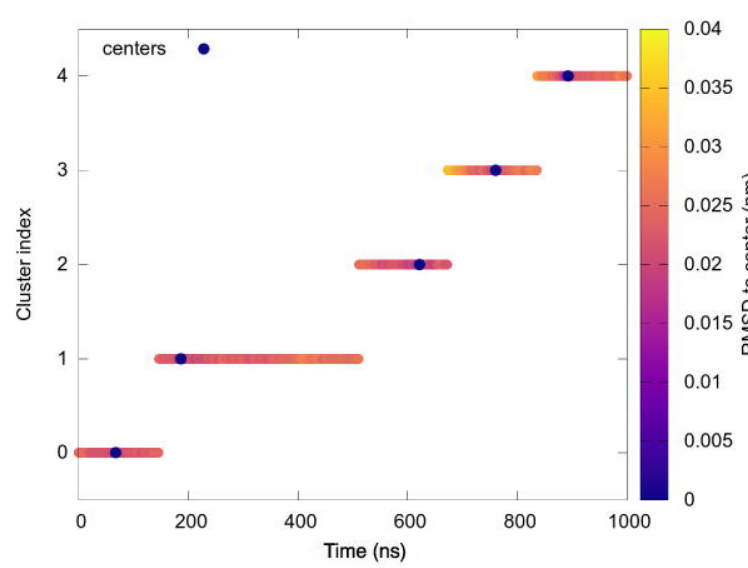

B $\beta 5-\alpha 2$ loop 


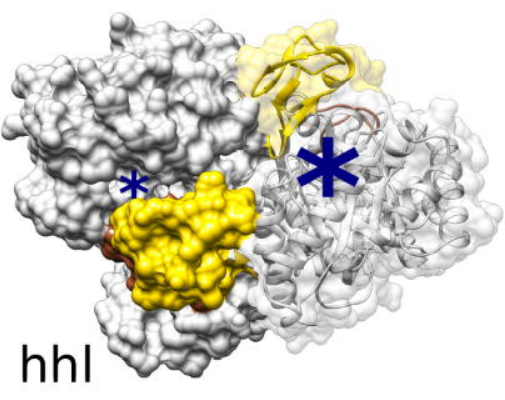

$\beta 5-\alpha 2$ loop
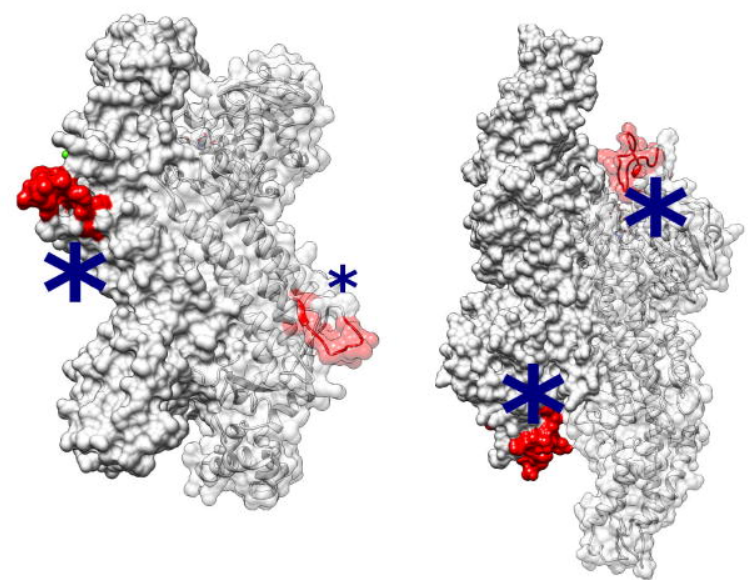

317-328 loop 102-124 loop

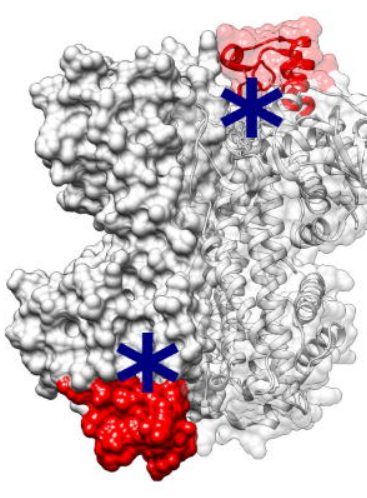

39-80 loop 